\title{
MÉTODOS QUANTITATIVOS NÃO SÃO A SALVAÇÃO DE TODA LAVOURA ETNOBOTÂNICA: O QUE DIZ JAVIER CABALLERO
}

\section{QUANTITATIVE METHODS ARE NOT THE SALVATION OF ALL ETHNOBOTANICAL CROPS: WHAT JAVIER CABALLERO SAYS}

\author{
Lin Chau MING ${ }^{*}$; Ari de Freitas HIDALGO²
}

1.Departamento de Horticultura, Faculdade de Ciências Agronômicas, Universidade Estadual Paulista, Botucatu, SP, e-mail: linming2809@gmail.com; 2. Departamento de Produção Animal e Vegetal, Faculdade de Ciências Agrárias, Universidade Federal do Amazonas, Manaus, AM, e-mail: afreitash @gmail.com

\section{RESUMO}

Submitted: 31/01/2020; Accepted: 19/02/2020

Este trabalho refere-se à entrevista feita pelos autores com o Professor Javier Caballero Nieto, da Universidad Nacional Autónoma de México (UNAM), sobre assuntos relacionados a métodos quantitativos em Etnobotânica, algumas de suas principais características, e sua complementariedade com enfoques qualitativos de pesquisa, tema que tem sido objeto de aprofundamentos nos últimos anos no Brasil (e no mundo). Javier Caballero é biólogo formado pela própria UNAM, em 1974, e fez mestrado na mesma universidade (1980) e também na Universidade da Califórnia, Berkeley (1988), onde também terminou o doutorado em 1994. Tem demonstrado interesse em Etnobotânica desde o tempo de graduação e depois se concentrou em temas mais ligados aos métodos quantitativos na área, mas sempre envolvendo uma relação sólida com aspectos ecológicos e de domesticação das plantas e as comunidades tradicionais. Suas opiniões ajudam a esclarecer vários aspectos sobre o assunto e também a aprofundar e estimular o debate entre os interessados na área.

Palavras-chave: etnobotânica, métodos quantitativos; Javier Caballero; UNAM

\section{ABSTRACT}

This work refers to the interview made by the authors with Professor Javier Caballero Nieto, from the Universidad Nacional Autónoma de México (UNAM), on subjects related to quantitative methods in Ethnobotany, some of its main characteristics, and its complementarity with qualitative research approaches, theme that has been the object of deepening in recent years in Brazil (and in the world). Javier Caballero is a biologist graduated from UNAM, in 1974 and obtained master degree at the same university (1980) and also at the University of California, Berkeley (1988) and where he also finished his doctorate (1994). He has shown an interest in Ethnobotany since graduation and later focused on themes more linked to quantitative methods in the area, but always involving a solid relationship with ecological and domestication aspects of plants and traditional communities. His opinions help to clarify several aspects on the subject and also to deepen and stimulate the debate among those interested in the area.

Key words: ethnobotany, quantitative methods; Javier Caballero; UNAM

\section{INTRODUÇÃO}

A Etnobotânica ainda é uma ciência considerada nova, comparada a outras. Sem dúvida, ainda está com pouco tempo de início e de maturação. Temos visto a cada ano importantes contribuições de professores, pesquisadores, estudantes e técnicos aqui no Brasil. Cada um se aprofundando em determinada área geográfica, população tradicional (e indígena), bem como em aspectos que ampliam os enfoques mais corriqueiros, mais comuns em sua maioria.

Mesmo assim, a Etnobotânica tem sido enquadrada como uma Ciência menor, principalmente por pessoas de outras áreas científicas. Falam que somos poucos, falam que nossos trabalhos são simples demais, falam que também são poucos os trabalhos publicados. E falam que em nossas pesquisas não são utilizados métodos quantitativos para análise dos resultados, para ter mais confiabilidade estatística. Essas críticas são recorrentes, como também assim o são o debate com os contrapontos de cada um dos itens. 
Temos superado algumas dessas críticas ao longo do tempo, mas havia a dificuldade na questão dos métodos quantitativos, pela quase inexistência de profissionais com experiência por aqui no Brasil. Estudantes de pós-graduação e alguns poucos professores se enveredavam na área, colocando um promissor cenário acadêmico. Contatos e relações acadêmicas com especialistas estrangeiros poderiam reforçar a área e melhor gabaritar os trabalhos por aqui desenvolvidos.

Conheci o Javier Caballero a partir de uma indicação do Miguel Alexiades, etnobotânico greco-espanhol que havia conhecido em Nova York, durante um estágio que realizei no Jardim Botânico daquela cidade, em 1996. Muitos falavam deste mexicano alto, professor da Universidad Nacional Autónoma de México (UNAM), lotado no Jardim Botânico do Instituto de Biologia dessa Universidade, na capital daquele país (Figuras 1 e 2), e que se destacava no cenário internacional por suas pesquisas envolvendo métodos quantitativos em estudos etnobotânicos.

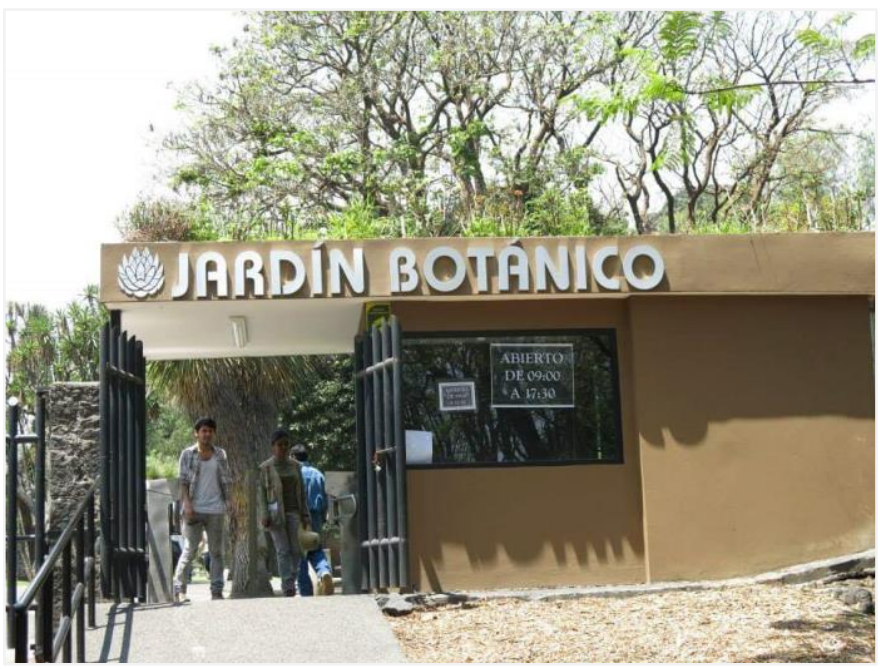

Figura 1. Entrada do Jardim Botânico do Instituto de Biologia, UNAM, México D.F. (Autor: Almecina Balbino Ferreira)

Tentei contatá-lo em 1997 quando participei do II Congresso Internacional de Etnobotânica em Mérida, México, ocasião em que aproveitei a viagem e fui para a Cidade do México visitar a UNAM (Figura 3), mas não consegui encontrá-lo. Hoje podem estranhar o fato de não ter havido o contato, a comunicação era bem mais difícil, mas, ao contrário de muitos na época, demorei a adotar os meios eletrônicos de comunicação virtual, tendo usado os e-mails apenas em 1998. Impensável nos dias atuais.

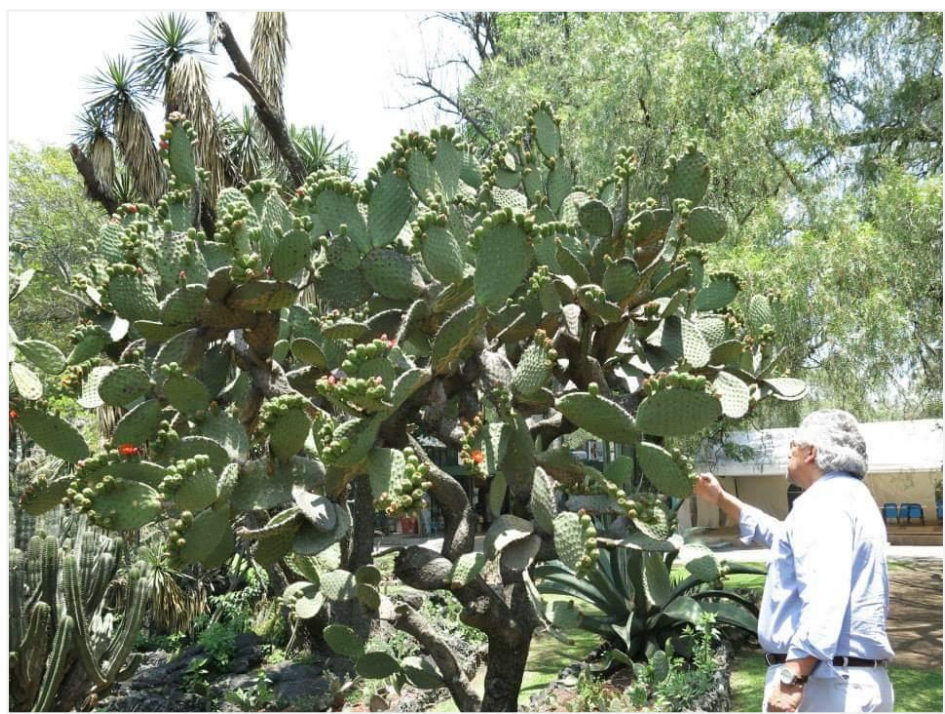

Figura 2. Javier Caballero ao lado de um belo exemplar de Opuntia no Jardim Botânico (Autor: Almecina Balbino Ferreira)

Mas a viagem valeu muito para participar do evento e também para visitar alguns locais interessantes na Capital, como a Praça Zócalo, no centro da cidade, onde tem esse painel de azulejo (Figura 4) mostrando as "tunas", símbolo da cidade; o Museu Nacional de Antropologia (Figura 5), essencial e imperdível para quem quer 
conhecer um pouco a história pré-colombiana e etnografia do povo mexicano; as pirâmides de Teotihuacan (Figura 6), a Catedral Metropolitana, os painéis de Diego Rivera nas paredes do Palácio Nacional (Figura 7), etc., etc..

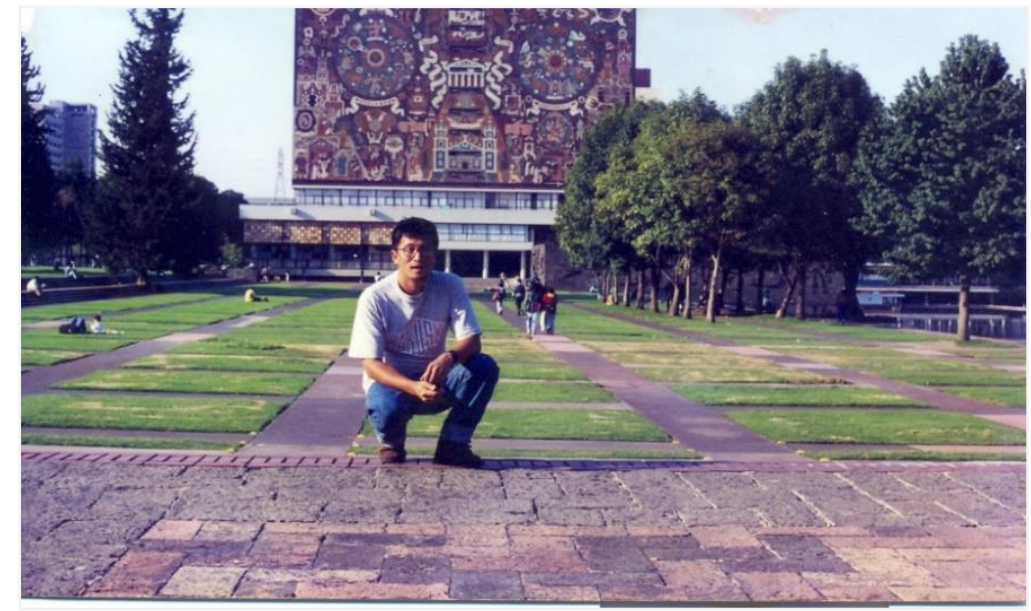

Figura 3. Visita ao campus da Universidad Nacional Autónoma de México, na capital mexicana (Autor: Lin Chau Ming)

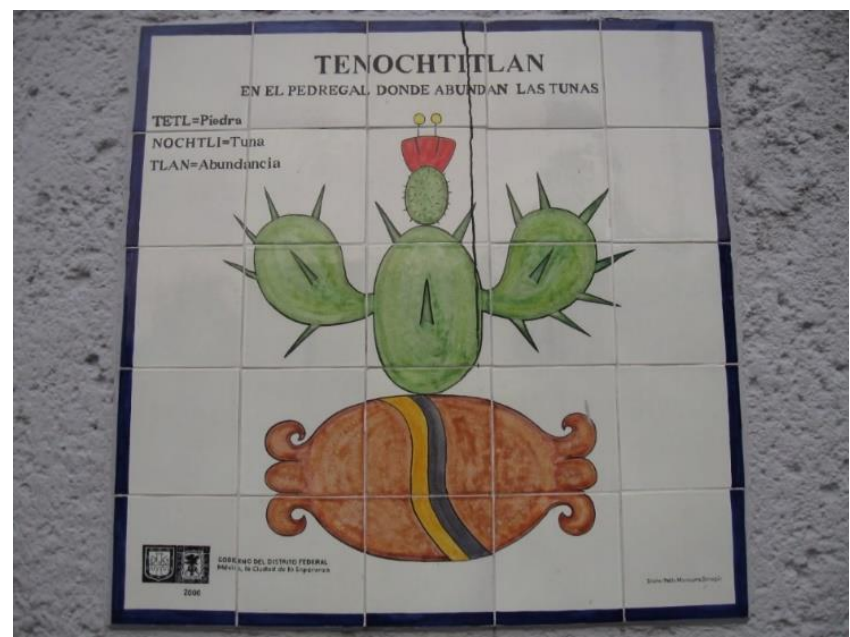

Figura 4. Painel de azulejo na capital mexicana, explicando origem do nome (Autor: Lin Chau Ming)

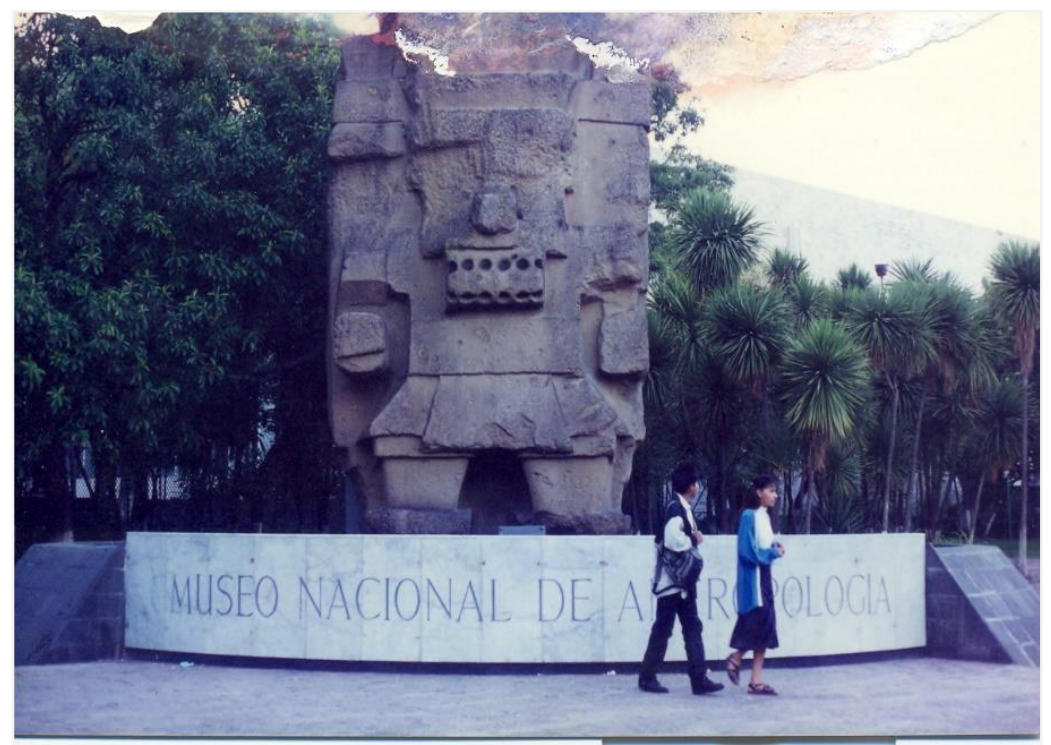

Figura 5. Entrada do Museu Nacional de Antropologia - Cidade do México. (Autor: Lin Chau Ming) 


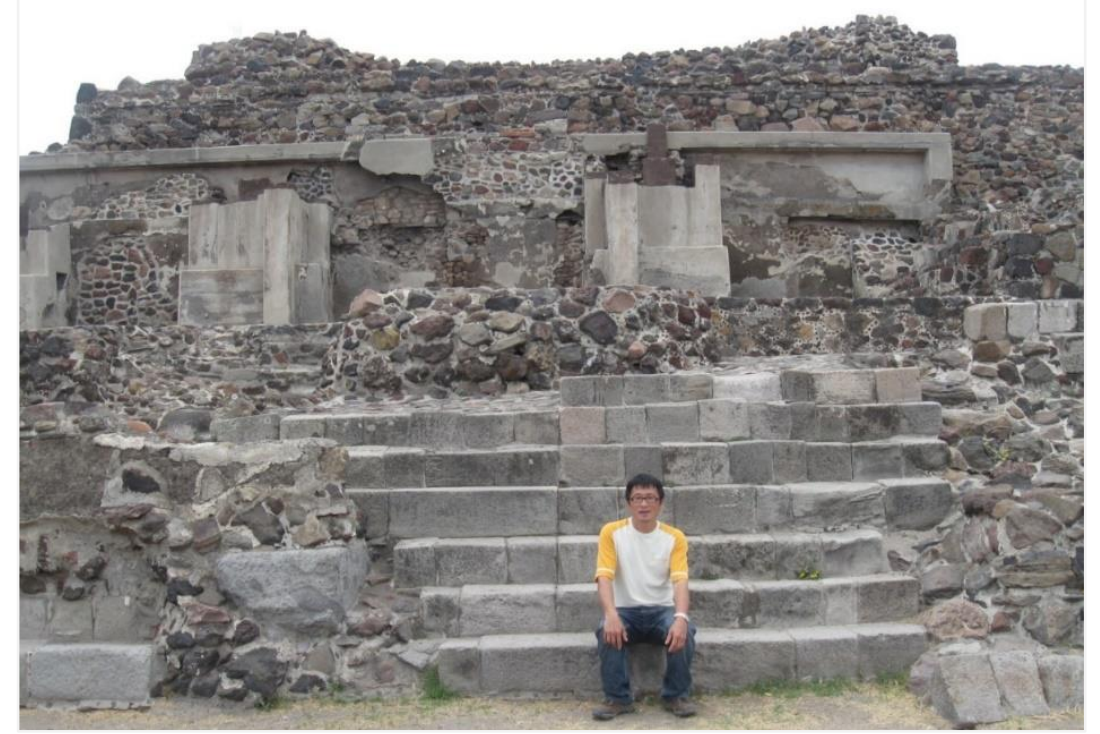

Figura 6. Templo do Milho, no sítio arqueológico das Pirâmides em Teotihuacan (Autor: Lin Chau Ming)

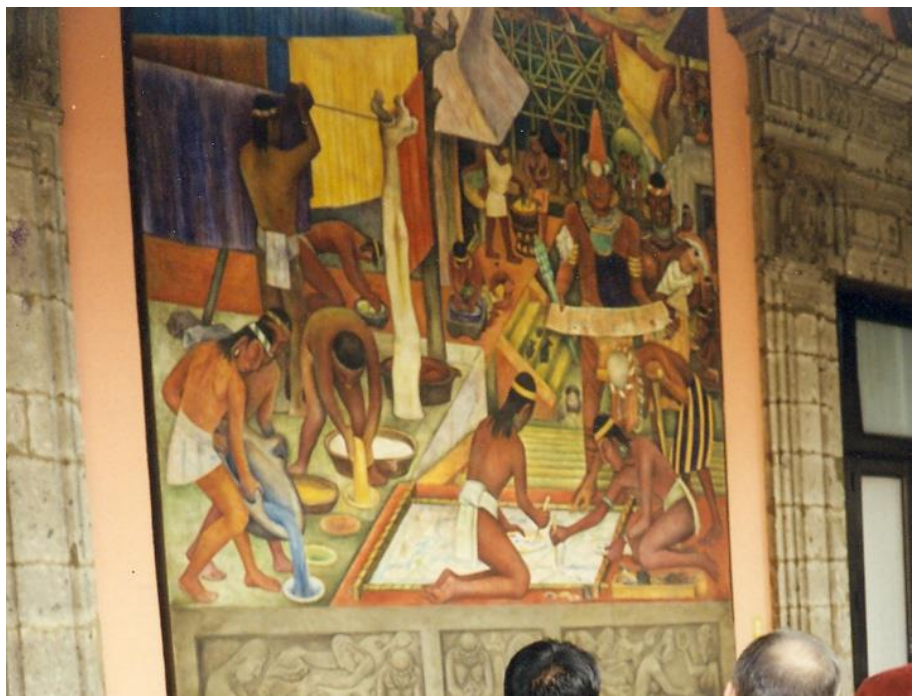

Figura 7. Painel de Diego Rivera sobre a história do milho, no Palácio Nacional, na capital mexicana (Autor: Lin Chau Ming)

Miguel, na época um estudante de doutorado orientado pelo etnobotânico americano Michael Balick, do Jardim Botânico de Nova York, preparava um livro sobre pesquisa etnobotânica e incluiria neste, capítulos com métodos quantitativos, e então estudou diversos trabalhos feitos pelo professor Caballero. E o recomendou.

Nessa época não havia sites acadêmicos onde era possível verificar os currículos dos pesquisadores, mas hoje, já com a existência deles, podem ser verificados seus inúmeros trabalhos etnobotânicos. Para confirmar, entre no Google Scholar e digite seu nome. Pronto! A indicação havia sido precisa.

Convidei Miguel para ministrar um curso sobre Etnobotânica, com conteúdo mais geral, e esse curso ocorreu um ano depois da vinda do Javier e que também foi bastante concorrido, com participação de mais de 20 estudantes e profissionais. Além do carisma, capacidade intelectual e forma de dar aula, um verdadeiro "Deus Grego", segundo os comentários da parte feminina dos participantes.

Então, Javier veio ao Brasil, a meu convite, em 1998, para ministrar curso sobre sua especialidade, os tais dos métodos quantitativos em Etnobotânica, que o pessoal da área no Brasil ainda tateava. Seria uma boa contribuição para o seu avanço. Ministrou o curso em Botucatu, com quase duas dezenas de alunos de pósgraduação de vários cantos do Brasil, de diferentes programas, ávidos por novas informações e trocas de experiência nessa área. Atualmente, muitos dos participantes do curso são professores ou pesquisadores na área. Tente identificar as pessoas na foto abaixo (Figura 8 e também na foto seguinte a ela, Figura 9, do curso do Miguel). 


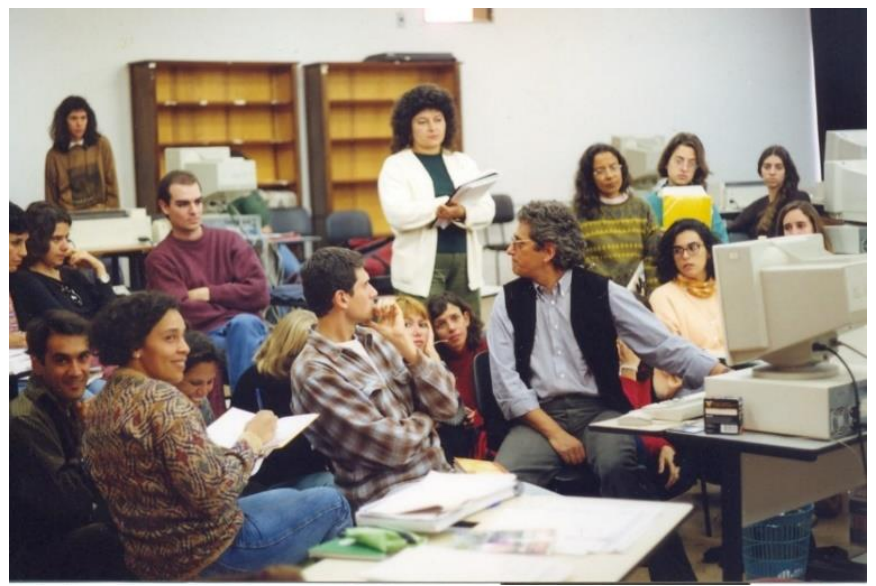

Figura 8. Javier Caballero (de colete escuro), na aula de Métodos Quantitativos em Etnobotânica, na Faculdade de Ciências Agronômicas, UNESP, Botucatu, 1998 (Autor: Lin Chau Ming)

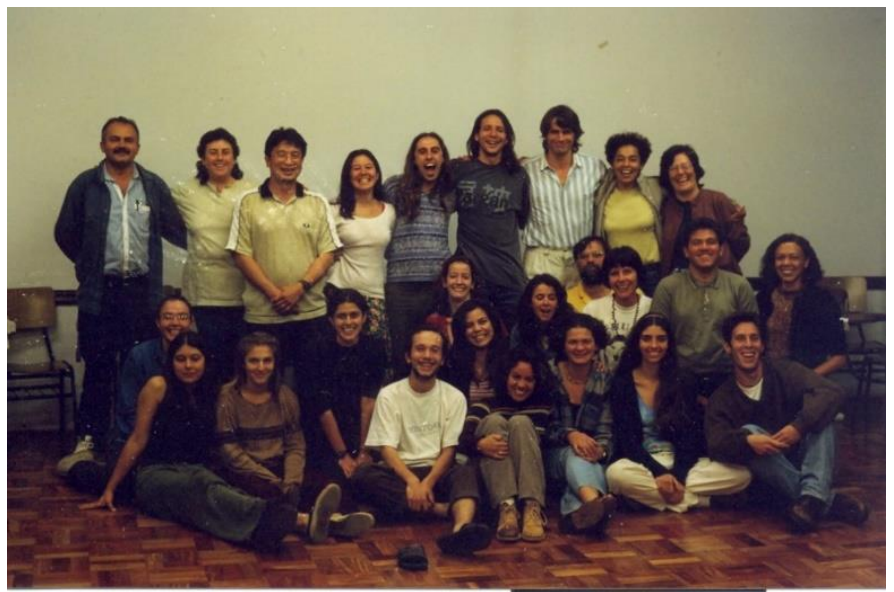

Figura 9. Miguel Alexiades (camisa com listras verticais, em pé) com os participantes do curso em Botucatu, 1999 (Autor: Lin Chau Ming)

Não tenho absoluta certeza, mas deve ter sido o primeiro curso sobre esse tema por aqui. Uma bela contribuição dada no Brasil e que sua estadia foi ainda mais bem aproveitada porque ele participou do II Simpósio Brasileiro de Etnobiologia e Etnoecologia, na cidade de São Carlos, logo após o final do curso em Botucatu.

Convidei-o novamente para outro curso em 2014, de novo na cidade onde moro. Ficou hospedado em casa, então pude conversar com ele mais longamente e também conhecer um pouco mais do cidadão Javier e não apenas do professor Caballero. Aceitou também receber estudantes de pós-graduação em atividades de pesquisa lá no México, ajudando a avaliar resultados de pesquisa quantitativa. Acabei por organizar um estágio para uma de minhas orientadas de Doutorado na época, Almecina Ferreira Balbino (Figura 10), hoje professora da Universidade Federal do Acre em Rio Branco. Javier tem como uma assistente, Andrea Martinez-Ballesté (Figura 11), também trabalhando com métodos quantitativos. Almecina teve ótimos acompanhamentos durante sua estada na capital mexicana.

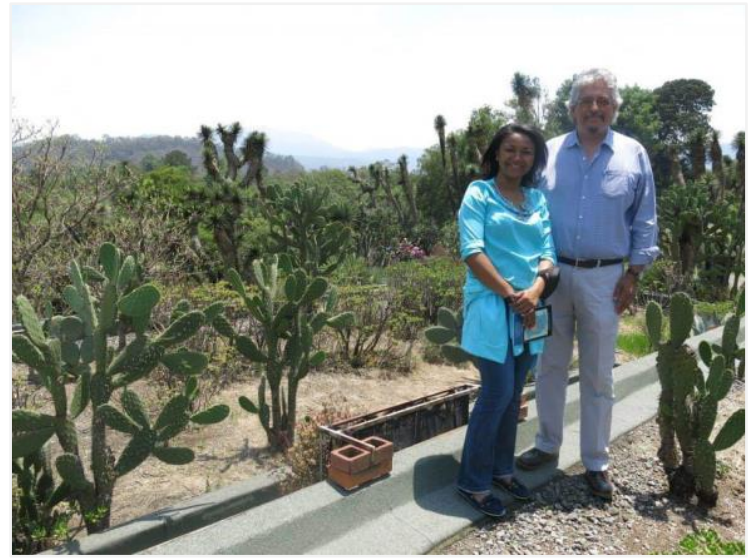

Figura 10. Almecina Balbino e Javier Caballero, na coleção de cactos do Jardim Botânico da UNAM - Cidade do México (Autor: Almecina Balbino Ferreira) 


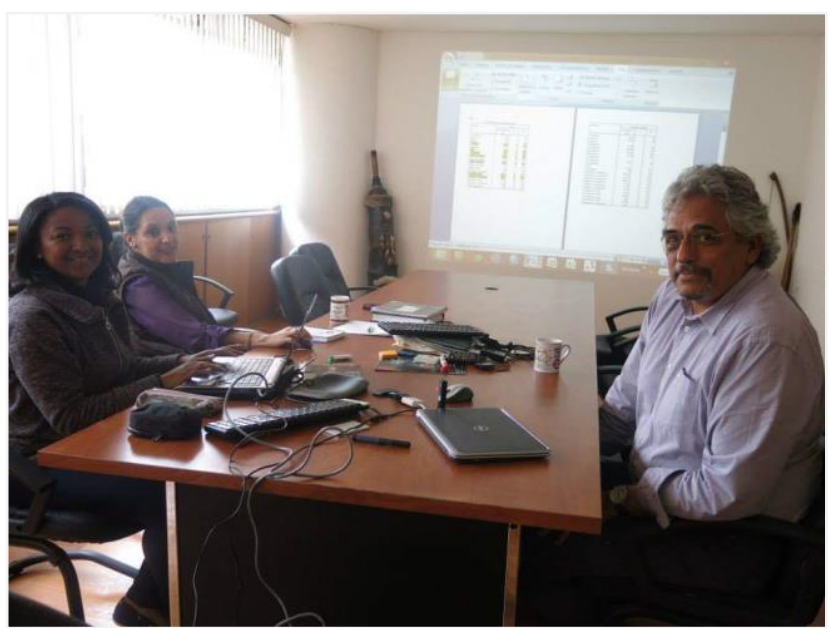

Figura 11. Da esquerda para a direita, Almecina, Andrea e Javier, no Jardim Botânico da UNAM, na Cidade do México (Autor: Almecina Balbino Ferreira)

Naquela ocasião estava em Botucatu para participar de uma banca de defesa de tese, o professor Ari de Freitas Hidalgo (Figura 12), da Universidade Federal do Amazonas, em Manaus, etnobotânico por excelência, com grande experiência de campo e que também, como eu, tinha várias dúvidas sobre essa área metodológica na Etnobotânica, e então eu aproveitei e o convidei para participar de uma entrevista com o Javier, na minha casa.

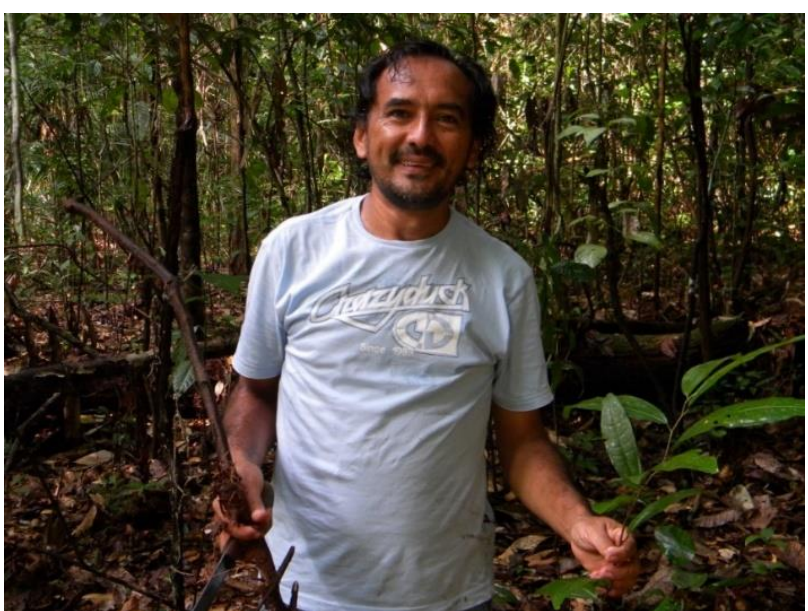

Figura 12. Professor Ari de Freitas Hidalgo, da UFAM - Manaus, coletando saracura-mirá (Autor: Ari de Freitas Hidalgo)

Aceitou e realizamos uma conversa rápida com o professor Caballero, pois este não tinha muito tempo disponível, dado o envolvimento no curso ministrado e vivo assédio que os alunos lhe impetravam (Figuras 13 e 14). A conversa nos prendeu bastante com a firmeza de suas colocações e argumentos (Figura 15). Tiramos com o professor Caballero muitas de nossas dúvidas teóricas e metodológicas, mas também ficamos com outras, mesmo durante o pouco tempo que tivemos para essa conversa. E do contato, ficaram marcadas a simplicidade e amizade demonstradas novamente. Em São Carlos, numa eleição realizada entre os participantes do simpósio, ele recebeu o título de "Mister Simpatia". Nada mais justo.

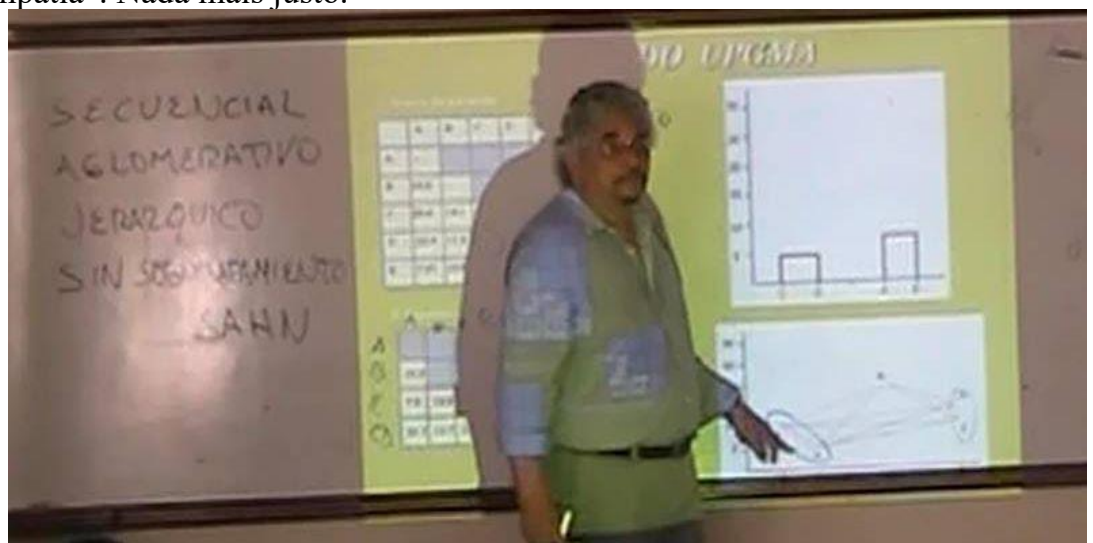

Figura 13. Javier em novo curso na UNESP - Botucatu (Autor: Daniel Garcia) 


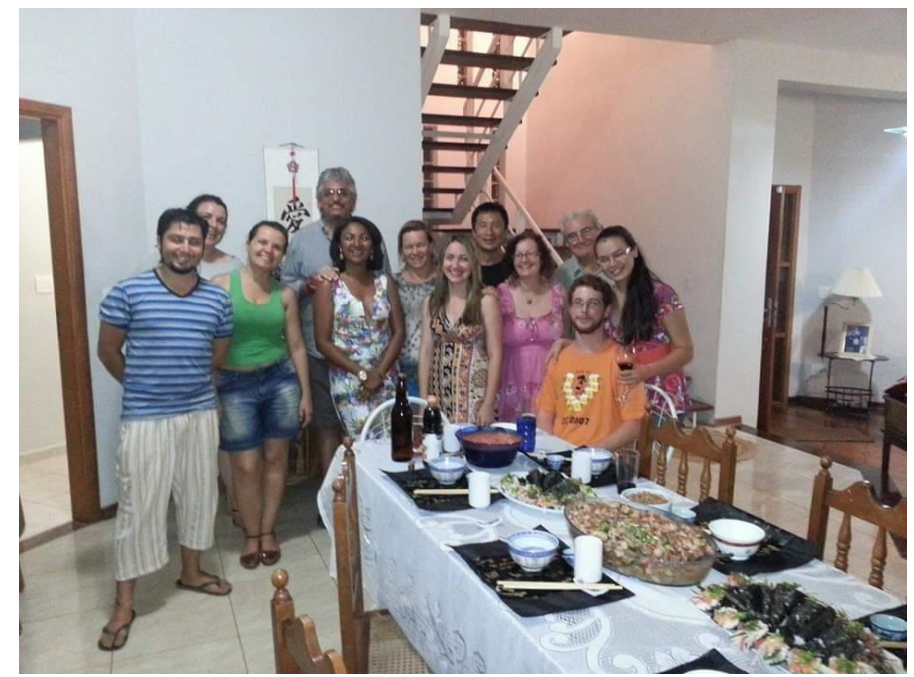

Figura 14. Javier em jantar de confraternização em Botucatu (Autor: Almecina Balbino Ferreira)

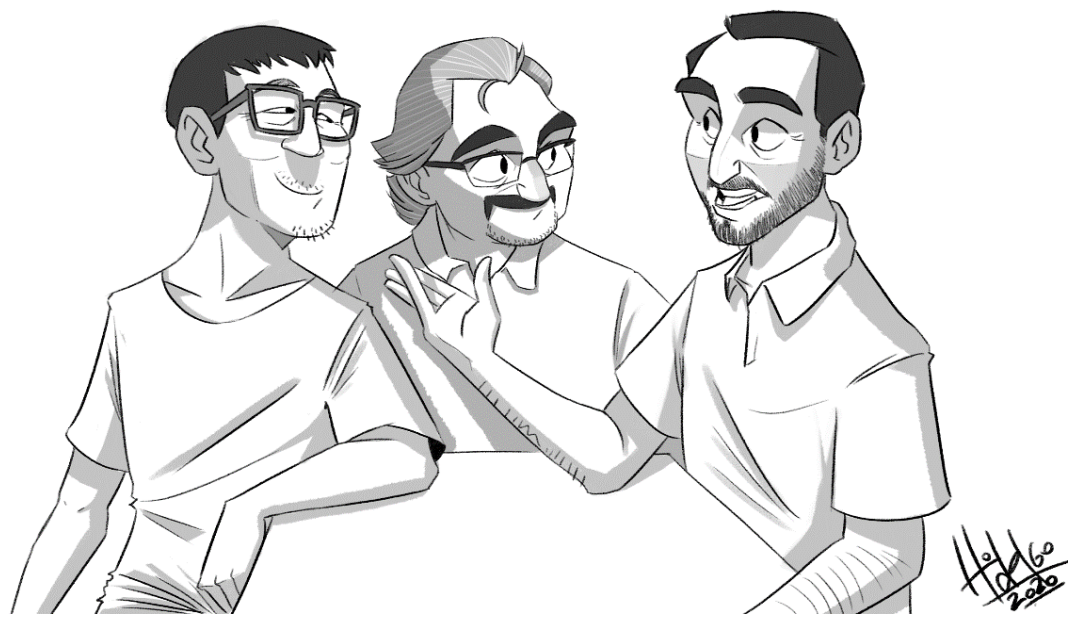

Figura 15. Lin, Javier e Ari, na conversa (Autor: Eduardo Moraes Hidalgo)

\section{A ENTREVISTA}

A primeira pergunta abordava uma questão ampla, sobre a importância que os métodos quantitativos têm nos trabalhos etnobotânicos nos dias atuais.

Javier - Yo creo que cada vez es mayor la importancia que tiene la aplicación de métodos cuantitativos para entender los fenómenos de la interacción entre los grupos humanos y las plantas, su entorno en general. Yo creo que la aplicación de métodos cuantitativos surge de la necesidad de formalizar nuestra disciplina como una disciplina científica, que puede ser que en el pasado no llegó a verse como una Ciencia. Hacer Etnobotánica era como hacer folklore y describir lo valioso que ella es sin ser reconocida como una Ciencia. El problema con la Etnobotánica, creo yo, es que es un campo de conocimiento muy complejo y que esta interviene en fenómenos culturales, sociales y biológicos y en diferentes órdenes. Yo creo que en el inicio eso trajo mayores dificultades más, al momento de aplicar métodos más rigorosos. Sin embargo, yo creo que los métodos cuantitativos no sustituyen en si su enfoque cualitativo. El maestro Miguel Ángel Martínez (http://www.ibiologia.unam.mx/gela/p-9.html; $\quad$ http://www.scielo.org.mx/scielo.php?script=sci_arttext\&pid=S018771512007000300001), fue un ilustre etnobotánico mexicano, que acostumbraba decir que lo primero que un etnobotánico debe hacer antes de iniciar una investigación es llegar al pueblo, pasear por la plaza... y que la gente lo mire, empezar a establecer relaciones con las personas locales y conversar! Y... solamente después de establecida esta relación es que uno obtiene la información de carácter cuantitativo, a través de las entrevistas abiertas, y la observación participante. Solamente cuando pasada esa etapa es cuando uno puede pensar en aplicar algún método cuantitativo para la obtención de datos que serán posteriormente analizados para luego a partir de allí pensar en probar alguna hipótesis... los enfoques cualitativos y cuantitativos no son opuestos.

Conforme dito, os métodos qualitativos e quantitativos não são excludentes, não. Já tinha essa afirmativa sendo trabalhada na cabeça há algum tempo, pois pensava que nem todos os etnobotânicos têm interesse em índices quantitativos e nem por isso seus trabalhos não são de qualidade. E a isso, Caballero respondeu:

- Son complementarios, no son excluyentes. Son complementarios y muchas veces primero debemos hacer un trabajo cualitativo, detallado y profundo para luego poder aplicar los métodos cuantitativos. Segunda pregunta: Hay algunas ocasiones en que un proyecto de investigación no los tiene y por tanto es necesario pensar en que... bueno... pueden aplicarse 
métodos cuantitativos, casi desde el comienzo de nuestra investigación, pero siempre tendremos que hacer un trabajo detallado con las personas locales.

Colocando essa situação, isso sempre nos preocupa pois poderia parecer, em nível mundial, uma consequência de poder haver um desprestígio. Das áreas de Ciências Sociais, Antropologia e o oposto acontecendo na parte de Biologia, pois tem muito mais pessoas trabalhando e então os governos e as instituições financiadoras, eles dão bem mais valor do que para a área de Antropologia, por exemplo. No Brasil a gente consegue ver uma grande diferença nos recursos injetados. E nas áreas de Antropologia e das Ciências Sociais não recebem quase nada e você acabou de comentar que a Etnobotânica era encarada como uma não-Ciência...

Javier - Exactamente... si!

- E imagina agora, a Etnobotânica volta a poder ser alguma coisa mais forte, a ser Ciência se tiver algum enfoque mais quantitativo, perguntei sem muito pensar...

Javier - Sí... yo creo que los enfoques cuantitativos nos ayudan a cambiar esta situación. Yo estoy de acuerdo que la tendencia es que disciplinas como la Antropología y la Etnobiología, han perdido interés y han perdido prestigio, por varias razones. Los procesos de globalización, nivel mundial, donde ya lo particular, lo local, no tiene ya mucha importancia. Entonces, el trabajo etnobotánico se ve como una curiosidad, como estudiar el folklore de algún lugar, de algún grupo de personas. Y lo que importa es, por ejemplo, en la Agronomía diseñar este... obtener variedades mejoradas de mayor valor comercial, ¿hum?! Y lo tradicional pierde... pierde interés. Pero, cuando la Etnobotánica estudia con enfoque científico, las creencias y los fenómenos de interacción creencias, es el momento en que realmente se muestra la importancia que todavía tiene, en recuperar el conocimiento tradicional de las populaciones locales. $Y$ transformarlo en formas de aprovechamiento de los recursos vegetales que sean sostenibles y que sean, ecológicamente sostenibles, y que sean económicamente redituables también para las mismas populaciones locales, y que en algunos casos también puedan tener un desarrollo económico a nivel de mercado nacional o internacional. Y eso frecuentemente se logra cuando nosotros hacemos una investigación más formal, que incorpora métodos de análisis formales. Yo no quiero decir que siempre tenemos que probar hipótesis estadísticamente en el campo. Pero sí, tenemos que hacer preguntas muy claras, tenemos que definir muy bien los problemas que queremos investigar. Los factores que intervienen en las interacciones entre los humanos y las plantas y como los métodos cuantitativos nos ayudan mucho para esto.

Ari interferiu nessa questão:

- Mas pode parecer que os métodos quantitativos sejam extremamente mais úteis em situações utilitaristas, em objetivos utilitaristas da pesquisa etnobotânica, principalmente com números e dar valores, daí parece que ressalta a importância.

Javier - Pero no, no... sí, sí, así es que se tornan importantes cuando hay enfoques utilitaristas, pero también hay otros problemas de interés de la Etnobotánica que no tienen una aplicación utilitaria y donde los métodos cuantitativos, la cuantificación, nos ayuda a entender mejor los fenómenos. Por ejemplo, en el caso, del cambio cultural... todos sabemos que los procesos de modernización económica, social, la globalización, generan cambio y la pérdida del conocimiento tradicional. Pero, más allá de eso lugar común sabemos poco como funciona. Y no podemos probar frecuentemente que eso está corriendo de esa manera. Sin embargo, la cuantificación nos ayuda a entender bien como operan estos fenómenos. Recientemente en México, con un alumno mío, Alfredo Saynes-Vasquez, realizamos un estudio muy interesante del proceso de cambio cultural entre los zapotecos del Istmo de Tehuantepec, de Oaxaca en México (https://journals.plos.org/plosone/article?id=10.1371/journal.pone.0151693), donde tenemos una situación en la que hay un cambio cultural que es distinto entre tres diferentes comunidades y donde están relacionados el factor de ocupación. Hay agricultores, pero también hay agricultores tradicionales, hay agricultores comerciales, hay empleados, obreros donde nosotros queríamos entender cómo ocurre la pérdida de conocimiento tradicional. Y en estos grupos, si hay diferencias, y en este proceso entre comunidades y al interior de las comunidades de acuerdo a la ocupación de las personas. Y es un análisis cuantitativo formal, en el que aplicamos modelos de análisis lineales no generalizados los cuales nos permitieron entender como ocurren los procesos en diferentes escenarios. Y por eso podríamos pensar que tiene una aplicabilidad, pero no necesariamente.

Lin - Não necesariamente ...

Javier - Utilitarista... o contrario... puede ser para diseñar estrategias que reviertan este proceso de cambio... de cambio cultural. $Y$, bueno, así puedes entender como ocurren estos fenómenos...

Lin - E os antropólogos concordam? Ou têm uma boa percepção sobre esses métodos?

Pensando um pouco, Javier responde:

- No... en general no... Los antropólogos no. No, porque eso es otra ... otra escuela de pensamiento. Y también depende de que antropólogos hablamos, ¿no?!

Ari - Mas no geral ....

Javier - Hay diferentes escuelas en Antropología...

Ari - Em geral há dificuldade de aceitar, não?!

Javier - Claro. Es que también en Antropología hay diferentes enfoques. Hay los que estudian la cultura, hay quienes piensan que la cultura es un fenómeno único, en el espacio y el tiempo, y que no es posible hacer generalizaciones universales. Y por lo tanto rechazan cualquier enfoque científico. Entonces, bueno... hay antropólogos que, sí, les parece que es importante desarrollar enfoques cuantitativos, pero hay muchos otros antropólogos que no. Hay... bueno, por ejemplo, hay un programa, de textos que yo uso en discursos con más frecuencia...

Lin - Hmmm... Quais? Pode citar algum exemplo?

Javier - Son textos escritos por antropólogos, con enfoques cuantitativos. Uno de ellos, Russel Bernard describe un artículo sobre métodos de investigación de campo en Antropología (http://nersp.osg.ufl.edu/ ufruss/documents/comparing\%20four\%20methods.pdf). Con enfoques estadísticos muy rigorosos. Pero, bueno, hay muchos otros antropólogos que... no... eso, no siempre.

Ari - Pensando assim, a ideia é trabalhar com recomendações para um jovem etnobotânico, né?! Para quem está iniciando. Mas eu acho que vai servir também para quem já tá no campo?! E ainda pensa muito qualitativamente. E como é 
questão do preparo, da ida a campo, esse preparo nas perguntas, para não chegar depois e tentar tirar dali uma coisa mais quantitativa, quando o enfoque foi totalmente aberto.

Javier - Sí... yo frecuentemente escucho estudiantes que me dicen, bueno, yo analicé mis datos de esto y esto manera, y tengo muchos datos. Y me preguntan por algún método de análisis que les diga algo. No... (risos).

Ari - Para tentar....

(Risos de todos...)

Javier - Lo primero... yo siempre les digo a los estudiantes, lo primero que tenemos que hacer es tener una pregunta de investigación clara, bien definida, definir los límites de nuestra investigación. Por supuesto tenemos que estar convencidos de que esa pregunta es relevante científicamente, no?! Si yo no puedo formular una pregunta de investigación como, por ejemplo, ¿cuantos granos de arena hay en una playa? ¿Qué relevancia científica tiene eso? Pero, cuando pensamos en la relación entre los seres humanos y su entorno vegetal, hay preguntas que son muy relevantes que tienen que ver con la percepción, el conocimiento que las personas tienen sobre estas plantas, su clasificación y la organización de ese conocimiento, las formas de uso, y sobre todo las formas de manejo. $Y$, bueno... porque... cualquiera de esos... son áreas de interés de la Etnobotánica, lo que tenemos que hacer es plantearnos una pregunta, claramente. ¿Qué es lo que yo quiero saber? Yo tengo esas observaciones, tengo estos antecedentes y yo tengo que definir qué es lo que yo quiero saber. Y yo_lo tengo que formular como una pregunta.

Esse é um ponto importante e nevrálgico e penso que muitos não conseguem enxergar, Assim, devemos destinar grande esforço intelectual para que isso possa ser entendido. Assim, perguntei: - Mas isso é válido para qualquer tipo de trabalho?

Javier - Cualquier tipo...efectivamente. Yo les digo a los estudiantes que la Etnobotánica es una Ciencia...

Lin - É...exatamente....

Javier - Como la Física, como la Química. Si nosotros desarrollamos enfoques científicos en Etnobotánica, podemos hacer lo mismo que hacen todos los científicos. Utilizamos un método bien definido. Empieza justamente al formular una pregunta de investigación. iY después les digo a mis estudiantes, bueno... tenemos la pregunta! Ahora lo que hay que pensar es cuál es la posible respuesta. Entonces, vamos a formular nuestra hipótesis de trabajo. ¿Qué es lo que ocurre en ese fenómeno? ¿Cuál es su relación entre los factores que a nosotros nos interesan? ¿Y les digo, bueno... esa hipótesis puede ser probada estadísticamente? ¿Cuantitativamente? Bueno, entonces, tendríamos que convertirla en una hipótesis estadística y tendríamos, entonces, después, que pensar que datos necesitamos. Después, como los vamos a obtener en el campo, y después, como los vamos a analizar. ¡Pero, lo más importante es después cómo vamos a interpretar esto! Ahí es cuando los estudiantes tienen alguna duda, es difícil pasar de las simples descripciones a la interpretación de los resultados que obtenemos. Pero, precisan un procedimiento lógico, ¿todas las... las Ciencias?, no! Solo que las preguntas que nos hacemos como etnobotánicos, son... yo creo que muy complejas... porque involucran fenómenos de dos órdenes muy diferentes, lo cultural y lo biológico. Hum?!

Ari - Javier, pensando assim, será que o fato de a gente se preocupar muito no quantitativo, por que no qualitativo a gente leva perguntas e a pessoa passa a informação, para aquisição de dados quantitativos eu não estaria mais direcionando a pergunta? A conversa? Eu não estaria quase que forçando a ter respostas? Quantificadas?

Javier - Sí, es..

Ari - E aí eu não alteraria a qualidade da informação?

Javier - Sí, es eso un riesgo. Nosotros, en nuestro interés de probar nuestra hipótesis de trabajo, podemos a veces inducir las respuestas en las personas que nosotros entrevistamos. Ahí, yo creo que... es el momento de dar las recomendaciones y decir lo que debe ser hecho, ¿no?! Primero, tenemos que elegir el método de entrevista más adecuado, tenemos que probar las preguntas de nuestra entrevista. iSi las entrevistas estructuradas sirven para eso! Debemos formular preguntas concretas que nos permitan obtener respuestas concretas para que podamos comparar las personas entrevistadas. Eso es el... eso sería una acción, claro, importante. Siempre tendremos el riesgo que nuestras preguntas no sean claras para las personas locales, o que por la manera en que las formulamos terminemos induciendo una respuesta. Bueno, yo creo que lo que hay que hacer primero es probar nuestras preguntas con nuestras personas y estar convencidos de que estamos controlando estos factores. Y después de eso, hay que diseñar un sistema de muestreo, que sea adecuado para la pregunta que estamos formulando. Si, nuestra pregunta tiene que estar relacionada con el conocimiento botánico de una comunidad, en general. Bueno, entrevistaremos a lo mejor solo jefes de la familia o solo hombres y mujeres, indistintamente si son casados, si son de una familia. Pero será mejor nuestra pregunta si logramos comparar el conocimiento de hombre, el jefe de la unidad domestica... y...el de la madre, de la.... bueno, entonces, tenemos que entrevistar siempre lo hombre y la mujer en cada familia. Si nuestra investigación tiene que ver con adquisición del conocimiento, probablemente... entonces, hay que entrevistar personas de diferentes edades... segunda pregunta $\mathrm{Si}$, nosotros tenemos que hacer un diseño experimental para la obtención de los datos. Como lo ha en Ecología.

Lin - Certo... isso sempre é necessário.

Javier - Los ecólogos usan experimentos en campo se en el que diseñan un experimento natural, que guía la manera como se van a obtener los datos. Y en el caso de la investigación etnobotánica el diseño lo hacemos en función de la pregunta. Vamos a decidir cuantas personas vamos a entrevistar, como las vamos a seleccionar, que les vamos a preguntar a todas, como vamos a registrar sus respuestas y como las vamos a comparar.

A tabulação das respostas sempre foi item que leva em conta os tipos de perguntas e então indaguei:

- Mas perguntas muito abertas fazem com que a gente tenha que adaptar as respostas ou fazer categorizações das respostas, pra depois poder tabular, né?!

Javier - Sí...

Lin - E em métodos quantitativos não se deve tanto fazer assim, a pergunta tão aberta assim?!

Javier - No...

Ari - Então, as estruturadas ficam... Mas a situação é essa, a gente está sempre talvez direcionando pra quantificar. Talvez a preocupação do morador, do entrevistado, não é com quantificação, com número... Ele quer a explicação dele, independentemente de valores a gente fica preocupado depois em transformar a explicação... 
Javier - Sí. Pero, también muchas veces la cuantificación, la comparación sistemática, esas respuestas nos llevan a hacer preguntas, entrevistas a profundidad, detalladas con un grupo pequeño de personas para documentar lo que vimos en una gráfica Por ejemplo, explicar mejor que es lo que pasa. Entonces, yo por eso digo que son complementarios los enfoques cuantitativos y cualitativos en la investigación.

Ari - E o qualitativo depois volta explicando os números.

Javier - Claro...

Lin - Esse grupo menor, da entrevista aprofundada, isso é um sistema de estratégia qualitativa? Porque em métodos sociológicos eles fazem isso aí, não?! Nas pesquisas dos programas eleitorais, eles pegam grupos pequenos e discutem pra ter orientações para o candidato, assim... mas sob o ponto de vista qualitativo.

Javier - Y otra cosa muy importante es la observación participante.

Lin - Dá pra fazer quantitativo com observação participante?

Javier - No... bueno, no es imposible, pero, implicaría que nosotros tuviéramos el control de lo que vamos a observar para que nos permita hacer una comparación. La esencia de los métodos cuantitativos es que nosotros vamos a entrevistar, o vamos a observar personas, plantas, lo que sea, y vamos a registrar datos similares que nos permitan comparar personas entre sí, o plantas entre sí. Eso es la esencia. Entonces, cuando hacemos observaciones participantes nosotros dependemos del fenómeno que estamos observando. Puede ocurrir de una manera o puede ocurrir de otra... Y esos son eventos únicos en el tiempo, ¿sí?!

Ari - Vai explicar depois...

Lin - Foge do controle do investigador, do pesquisador!

Ari - Ainda mais se ele aplicou, ele observou. A observação feita ajuda entender a resposta, depois explicar o que os números mostram, né?!

Lin - E complementarmente....

Ari - Complementarmente...

Javier-Complementariamente, sí... sí...

Ari - Mas eu acho aí por si só não consegue, não, né?! Observando...

Javier - No. Algunas veces el análisis cuantitativo de los fenómenos es suficiente para explicar un fenómeno, pero para entenderlo completamente en su base cultural, hay que complementarlo con entrevistas a profundidad, con observación participativa para recordar, aquello que decía uno de los antropólogos más famosos "el principio del desarrollo antropológico es desarrollo". Decir que los esquimales no comen en la canoa, cuando van en el mar no es suficiente, ;yo tengo que explicar es porque no lo hacen! Entonces, ese tipo de información ya los métodos cuantitativos no pueden explicar, pueden a lo mejor si, después de una serie de observaciones, o entrevistas, pueden decir, bueno, los esquimales de tal pueblo acostumbran comer en la canoa y los de este otro pueblo no. ¡Pero, no nos explica porque no lo hacen!

Lin - Não comem?

Javier - No comen nada. No comen ningún alimento mientras están navegando...

Lin - Ah, enquanto estão na água?!

Javier - Mientras navegan en una canoa. Si?! Eso tiene que ver con los mitos, con la interpretación del mundo de ese grupo de gente. Y eso, porque los métodos cuantitativos, no los explican.

Lin - Mesmo que ainda a gente tenha diferentes tipos de exemplos, para justificar os métodos qualitativos. Ainda aqui no Brasil muita gente acusa a Etnobotânica, pelo fato de não usar os métodos quantitativos, de ser uma Ciência menor.

Javier - Sí... eso pasa no solamente en Brasil, eso pasa en muchos otros países. En México, por ejemplo, años atrás, sobre todo los ecólogos, veían la Etnobotánica como una disciplina menor porque no tenía un desarrollo científico formal. Y hoy en día nuestros estudiantes, que hacen Etnobotánica, tienen un igual conocimiento de estadística, tienen capacidades para formular trabajos científicos y los resultados son comparables a los que obtienen cualquier estudiante en uno estudio ecológico. Y creo que la percepción que nuestros colegas de la ecología tienen ahora, sobre la Etnobotánica, ha cambiado, notablemente. Y pienso que eso ocurre a nivel internacional también. Solo que hay quien dice que se van al otro extremo. $Y$ piensan que si uno no calcula una chi-quadrada, no sirve de nada el trabajo, ¿no?! No...

Lin - (risos).

Javier - Yo creo que hay que entender la complejidad de nuestra disciplina y tomar las decisiones adecuadas. A mí, me parece, por ejemplo, que textos que narran la experiencia del campo son tremendo importantes, porque nos dan información de grande cualidad. Consiguen explicar la naturaleza de los fenómenos de interacción humanos-planta.

Javier Caballero se formou em Biologia em 1974 na própria UNAM e depois, em 1980, fez mestrado também na UNAM e outro na Universidade da Califórnia, em Berkeley (1988). Depois terminou o Doutorado na mesma instituição americana, em 1994. Os trabalhos dele envolveram Ecologia e Etnobotânica, e seu trabalho de conclusão de curso de graduação teve o título: "El ecológico del uso de la Tierra em um ejido del Trópico" e o de doutorado foi: "Use and manegement of Sabal Palms among the Maya of Yucatan". Pode-se ver que seu interesse em Etnobotânica já era expresso desde o tempo de graduação e que depois se concentrou em temas mais ligados aos métodos quantitativos na Etnobotânica, mas sempre envolvendo uma relação sólida com aspectos ecológicos e de domesticação das plantas e as comunidades tradicionais, como é possível ser verificado em seu currículo obtido pelo Google Scholar. Assim, fiz essa pergunta:

Lin - E na tua vida acadêmica, Javier, você começou diretamente assim, na pós-graduação, com métodos quantitativos?

Javier - No realmente, pero siempre he tenido un gusto por la formalización del trabajo. Cuando... hace mucho tiempo, yo hice mi investigación del grado y yo hice un estudio cuantitativo del balance energético de la agricultura de roza, en México. Utilizando los modelos que estaban muy comunes en ese tiempo, sobre todo entre los antropólogos norteamericanos, que analizaban las prácticas de agricultura de roza. Y con esto, esto tipo de enfoques si mostraban que eran eficientes. Cuando en ese tiempo mucha gente decía que la agricultura de roza era una práctica primitiva, que destruía la selva. Que era una práctica propia de la pobreza. Y estos enfoques mostraban que también había una lógica en este sistema. Entonces, si, desde el principio...

Lin - Desde o início... 
Javier - Yo tenía esta ... esta inclinación. No he hecho cuantificación toda mi vida, pero sí. Sí, tenía este interés por enfoques más formales, más científicos. Y yo creo que por eso es que cuando leía aquél trabajo de Philips \& Gentry (https://link.springer.com/article/10.1007/BF02862203), que proponen que hay que desarrollar métodos de cuantificación. Yo pensé, sí, tienen razón, esto es lo que tenemos que hacer, en Etnobotánica. Pero, al mismo tiempo, parte de mi formación es en Antropología, entendiendo el fenómeno cultural. Simpatizo mucho por los enfoques interpretativos en el estudio de la cultura. Esos que rechazan, la Ciencia. Mi parecen también muy poderosos para explicar fenómenos. Pero, sigo teniendo una predilección por los enfoques científicos.

Ari - Sim... não que o qualitativo não seja científico?!

Javier - No... no necesariamente no sería científico. Yo creo que si se puede hacer Ciencia sin números, en este proceso científico de definición del problema, de formular una hipótesis de trabajo, de reunir evidencias. No en forma estadística, pero sí, que los argumentos, las evidencias sean suficientemente sólidas, como para apoyar o rechazar una hipótesis de trabajo. Y que la interpretación y lo que sí está queriendo probar sea una interpretación válida. Que este... que en ese... en el marco de tu pregunta de investigación. ¿¿A veces los estudios descriptivos simplemente describen y describen sin un argumento lógico, hum?!

Ari - E, às vezes divaga muito também. O quantitativo direciona mais.

Javier - Exactamente... sí... Yo creo que sería una de las ventajas de cuantificar. Pero también se corre el riesgo, como yo dije antes, estudiantes que dicen es que ya hice veinte tablas diferentes (risos). Y.... bueno, ya no sé cómo concluir de esto. No, porque no hay una pregunta clara que direccione el análisis de esos datos.

Lin - Quando um aluno que quer trabalhar com a parte qualitativa vai conversar com você, você aceita ele?

Javier - Sí, su pregunta es absolutamente relevante, sí. Parte de un planteamiento teórico relevante. Sí, claro que sí. Creo que es un reto. Lo que yo siempre les digo a los estudiantes es cuál es tu pregunta.

Lin - Qual é a sua pergunta.

Javier - ¿Cuál es tu pregunta? Y yo encuentro que los dos... las dos situaciones, una en que aunque que se tenga una orientación cuantitativa no tiene una pregunta clara, y no saben qué hacer con la información que tienen. Pero hay otros que no pueden cuantificar sus datos, pero... pero su pregunta es clara. Entonces, creo que eso puede ser más útil que tener muchos datos e no tener una pregunta clara.

Lin - Sim, né?! Melhor que nada!

Javier - Claro, lo peor es si alguien no tiene una pregunta clara y solamente tiene puras descripciones anecdóticas de las cosas. Porque no... no tiene, (risos) muchas posibilidades de explicar lógicamente algún fenómeno.

Lin -É... depois eu vou querer que você me fale também, Javier, sobre se há um mínimo, não? De alguns dos índices, que devem ser ou que poderiam ser usados, assim, em estudos etnobotânicos quantitativos. Porque é claro que existem diferentes métodos e que nem todos são aplicáveis em qualquer situação. Mas o que você acha, assim, mais imprescindíveis, mais importantes?

Javier - Sí. Yo creo que todo estudiante en Etnobotánica debería de saber que es una media, que es la desviación estándar, y que es una probabilidad de medias, como se hace una Anova y lo que representa. Eso lo aprendemos todos en nuestra formación académica. No hay que dedicarse a la Etnobotánica para poder aprender eso. Pero ya, una vez trabajando en Etnobotánica, sobre todo el tipo de datos que obtenemos en nuestras investigaciones, que son conteos, son proporciones. $Y$ las pruebas que un estudiante deberá aprender son, como hacer una prueba de chi-cuadrada. Yo enseño en mis cursos que después de hacer una prueba de chi-cuadrada, nosotros podemos aplicar una prueba que no muchas personas conoce que se llama métodos ajustados de... métodos de residuos ajustados de Haberman (http://www.portalaction.com.br/tabela-decontingencia/analise-de-residuos) que nos dicen, por ejemplo, una chi-cuadrada nos dice que hay diferencias en el número de plantas que conocen los hombres y las mujeres, las diferencias son significativas. ¿Pero, nosotros como etnobotánicos, frecuentemente queremos saber quién sabe más? Y quién sabe... ¿Quién conoce más y quién conoce menos? Entonces, podemos aplicar una prueba como la de residuos ajustados de Haberman al resultado de chi-cuadrada y entonces obtenemos más información. $O$, por ejemplo, frecuentemente en Etnobotánica nosotros tenemos datos como conteos, contajes. ¿Cuántas plantas comestibles se usan en una comunidad? ¿Cuántas plantas comestibles... ¿cuáles especies de plantas comestibles se usan en nuestra comunidad? Y queremos investigar las diferencias, porque a lo mejor pensamos que hay procesos diferentes que se expresan en diferencias en número de plantas utilizadas. Entonces, una prueba de... se llama prueba de rango o de Mann-Whitney (http://www.leg.ufpr.br/lib/exe/fetch.php/disciplinas:ce001:vivian_-_teste_u_de_mann-whitney.pdf e http://www.inf.ufsc.br/ vera.carmo/Testes_de_Hipoteses/Testes_nao_parametricos_Mann-Whitney.pdf) , eso es una prueba muy adecuada para este tipo de datos. En mis cursos, yo enseño también métodos multivariados, porque frecuentemente nosotros queremos hacer tipologías de productores, de personas, seguro que conocen o usan. Entonces, los métodos multivariados son muy útiles, no nos permiten probar hipótesis, pero sí nos permiten explorar los datos y descubrir patrones de variación. En estudios de domesticación, por ejemplo, los métodos multivariados son muy importantes porque nos descubren patrones, tendencias, en la variación que hay entre las plantas, en las plantas como resultado del manejo por los agricultores, en manejo y la selección. $Y$, bueno, hoy en día, se emplean cada vez más y, con mayor frecuencia, estos modelos que se llaman modelos lineales generales, que tienen variantes para cuando tenemos datos que no son continuos, que no son variables como numéricas, no son variables cuantitativas continuas, son también datos como conteos. Entonces, compartes los modelos, hay los modelos log lineales, también llamados modelos lineales no generalizados, que nos dan mucha información, porque nos dicen cuál de los factores es el que nosotros estamos identificando ¿Que influye en el conocimiento el uso de la planta? ¿Cuáles son los factores más significativos? ¿Y cuál es la interacción entre los factores? Estos métodos nos dan respuestas a las preguntas que, como etnobotánicos, frecuentemente nos hacemos en el campo. Estos métodos apenas empiezan a utilizarse en Etnobotánica, pero, creo que en algunos años van a ser muy generales, van a ser muy comúnmente utilizados. Entonces, yo diría que los etnobotánicos tienen que aprender sobre estos métodos. Esto no quiere decir que todo los etnobotánicos se vuelvan estadísticos, se vuelvan especialistas en estadística. Hay que tener un conocimiento intuitivo, saber qué métodos podrían decirme que cosas, y, pues muchas veces será necesario ir a consultar un estadístico que nos ayude a consultar, a procesar nuestros datos. Y esto que dije es muy importante, previamente...

Ari - Previamente... 
Javier - Frecuentemente llegamos con los estadísticos y le ponemos nuestras series de datos y decimos ¿cómo los analizo? No es que quiere saber (risos).

Javier - Este... como los obtuviste. Entonces, uno a veces piensa que tiene que regresar al campo, empezar nuevamente la investigación.

Lin - Uma dúvida muito frequente é a questão da amostragem. E se a gente estabelece um número, a partir do tamanho da comunidade, aí vai ter aquela tabela com os números mínimos de entrevistas. Que muitas vezes isso não se consegue fazer, nós não conseguimos....

Ari - É...

Lin - De uma população de 200, entrevistar cento e quarenta e pouco...

Javier - Ya...

Lin - Então, isso na estatística ela pode ser entre aspas, assim, superada. Ou seja, nós podemos fazer métodos quantitativos, mas não exatamente atendendo o número mínimo de da amostra, né?!

Javier - Hã hã...

Lin - O número mínimo da amostra. Isso causa algum tipo de problema muito sério, nos tratamentos estatísticos?

Javier - (risos)

Lin - E como resolver? Já que no campo e na prática, não vamos conseguir entrevistar tanta gente assim?

Javier - Sí. Esto es un problema que frecuentemente me preguntan los estudiantes, siempre tenemos esta preocupación de que tamaño debe ser la muestra. ¿Cómo es que yo voy a trabajarla? Entonces, yo les muestro una tabla que viene en libro este de Russel Bernard (http://www.cycledoctoralfactec.com/uploads/7/9/0/7/7907144/\%5Bh._russell_bernard\%5D_research_methods_in_anthropol _bokos-z1___.pdf), donde compara el tamaño de la población y el tamaño de la muestra requerida. Entonces, se encuentra que para una populación de cincuenta, digamos, cincuenta personas, cincuenta familias, cincuenta huertos, nosotros deberíamos muestrear cuarenta.

Lin - É.

Javier - Y en el trabajo etnobotánico, sobre todo en regiones como el Amazonas... (risos)

Ari: - Não tem... (risos)

Javier - Donde, para muestrear una familia y luego a otra tienes que caminar mucho tiempo, y tienes tiempo limitado para la investigación, recursos limitados, tienes que tomar decisiones prácticas. Y Russel Bernard, como es un antropólogo, sabe muy bien sobre esto y esa es para mi una recomendación de oro. Y dice: alguna información es mejor que nada. Entonces seamos realistas, si no podemos muestrear de acuerdo a los requerimientos estadísticos, bueno, pues hagamos una muestra que, por lo menos, nos permita hacer un gráfico y probar estadísticamente una hipótesis. Entonces, yo les digo a los estudiantes, bueno, la población es de ochenta quintales? ¿Qué tamaño puedes escoger para tu muestra? 20, 30, es de un buen tamaño. No cumple los requerimientos de muestreo estadístico, pero con eso puedes hacer un gráfico, este es lo que te permite establecer relaciones entre los factores de esto fenómeno. $Y$, bueno, nosotros hemos publicado ya numerosos trabajos donde el muestreo no cumple los requerimientos estadísticos, pero el trabajo de campo está muy bien detallado, tan laborioso, pero que es imposible cumplir los requerimientos. o resultado.

Ari -E aí eu acho que aumenta o peso do enfoque qualitativo, que ajuda a explicar aquela amostra pequena, a entender

Javier - Claro. Claro. Pero lo que si no es posible es que tú quieras explicar el manejo de los quintales a partir de una entrevista a una única familia que tiene un quintal. Eso no es posible. Porque, uno de mis profesores decía que hacerlo a lo simple es poco más que periodismo, ¿no?! Cuando lo que tienes que hacer es Ciencia. Entonces, hay que probar las cosas y para eso necesitas una muestra que te permita comparar. Sobre todo para que tengamos una muestra lo más representativamente posible que nos permita hacer extrapolaciones al fenómeno que queremos investigar, ¿no?!

Lin - E Javier, mais um assunto, pode ser? Como é que você vê as tendências atuais, dos métodos quantitativos dentro da Etnobotânica?

Javier - Yo creo que cada vez es más claramente reconocida, la importancia de aplicar estos métodos. Aunque, creo que al mismo tiempo hay mucha resistencia. Hay mucha resistencia de etnobotánicos que toda su vida han hecho un trabajo más etnográfico, más descriptivo, y que si... a lo mejor sienten amenazada, amenaza su propio este... figura como etnobotánicos y se oponen al uso de estos métodos. Piensan también que a lo mejor muchos de ellos nos ven, a los que usamos cuantificación como personas arrogantes, como si despreciáramos el trabajo que ellos hacen. Entonces, hay una reacción de rechazo y a veces de enojo. Pero, yo creo que la tendencia es que, sobre todo si pensamos en jóvenes, la tendencia es que sí, cada vez se formaliza más el trabajo de campo y el trabajo de análisis de los datos, donde la cuantificación es un... es un elemento importante.

Lin - Ou seja, ainda é fazendo com que a parte qualitativa, continue menos valorizada, não?

Javier - Yo creo que la parte cualitativa tiene que ser una parte bien valorada. Pero, no debe ser excluyente, de la parte cuantitativa. Yo insisto que un trabajo detallado de entrevista, de observación participante, es un componente muy importante. Sí, y siendo un componente esencial de la investigación etnobotánica. Pero, no debe limitarse a eso, sí?! Yo creo que debe haber siempre una complementación, a veces por personas diferentes a lo mejor, trabajando en un mismo fenómeno, no?! ¡Y yo no sé si yo en este momento de mi carrera académica pasaría dos años en una comunidad haciendo observación participante... no! i¿Yo creo que ya no... a me gustaría que otras personas lo hicieran y que pudiéramos combinar los enfoques para explicar mejor los fenómenos, hum?!

Lin - Certo, Javier, há espaço para diferentes tendências. Agora a gente tem outra questão, a de que existem trabalhos etnobotânicos em que a amostragem foi uma única pessoa. Pode falar um pouco sobre isso?

Javier: - Hum.. hum..

Lin - E estatisticamente eu acho que acaba tendo muita dificuldade pra que isso seja aceitável, mas em métodos qualitativos isso pode por que pode ser utilizada a estratégia de história de vida...

Javier - Exactamente sí. Yo creo que si yo encuentro un estudio de plantas medicinales o plantas comestibles, hecho a partir de una sola persona, de un solo informante?! Pues yo no lo tiraría a la basura . (risos). No, yo no, para mí eso sería 
un estudio muy importante. El problema es que no se pueden hacer generalizaciones a partir de eso. ¿Sí?! Si yo no puedo hablar de.... ¿¿no sé, si es un estudio del uso medicinal de las plantas entre los Aymara y solamente fue entrevistada una persona?! Yo no diría que eso representa el conocimiento de plantas medicinales de los Aymara! Es una historia de vida que nos da elementos para analizar, pero no podemos hacer una extrapolación.

Ari - Não tem elemento de comparação! Não tem referencial.

Javier - Exactamente... sí! Es una historia de vida.

Ari - Referencial de si mesmo, não se compara.

Javier - Sí.

Lin - Javier, vamos encerrando a entrevista. Queria agradecer pela tua participação aqui nessa nossa conversa.

Javier - Muchas gracias. Al contrario, es en verdad una distinción poder practicar con ustedes sobre esto.

Lin - Agradecido. Muito Agradecido!

Ari - Muchas gracias

Todos riem.

Lin - Cinquenta minutos! (observando o relógio).

Javier - ¡Cincuenta minutos! ¡Y no más!

Todos riem de novo.

\section{AGRADECIMENTOS}

Os autores agradecem a Daniel Villamil Montero e Juan David Solano Mendoza, pela revisão do texto na parte em espanhol e a Douglas Charles Daly, pela tradução do título para o inglês. 